\title{
tic\&société
}

Vol. 9, No 1-2 | 1 er semestre 2015 - 2ème semestre 2015

Les religions au temps du numérique

\section{La foi catholique et les dispositifs numériques : le cas de la diaspora catholique vietnamienne}

Anh Ngoc HOANG

\section{(2) OpenEdition \\ Journals}

\section{Electronic version}

URL: http://journals.openedition.org/ticetsociete/1935

DOI: 10.4000/ticetsociete. 1935

\section{Publisher}

Association ARTIC

\section{Electronic reference}

Anh Ngoc HOANG, « La foi catholique et les dispositifs numériques : le cas de la diaspora catholique vietnamienne », tic\&société [Online], Vol. 9, № 1-2 | 1er semestre 2015 - 2ème semestre 2015, Online since 19 May 2019, connection on 10 December 2020. URL : http://journals.openedition.org/ ticetsociete/1935; DOI : https://doi.org/10.4000/ticetsociete.1935 
tic\&société - 9 (1-2), 2015

La foi catholique et les dispositifs numériques :

le cas de la diaspora catholique vietnamienne

Anh Ngoc HOANG

Université catholique de l'Ouest (UCO)

3, place André Leroy, BP 10808,

49008 Angers cedex 01

anh-ngoc.hoang@uco.fr 
La foi catholique et les dispositifs numériques :

le cas de la diaspora catholique vietnamienne

\section{Anh Ngoc HOANG}

\section{La foi catholique et les dispositifs numériques : le cas de la diaspora catholique vietnamienne}

Maître de conférences en sciences de l'information et de la communication à l'Université catholique de l'Ouest (UCO), Anh Ngoc HOANG mène un examen critique des transformations sociétales contemporaines liées aux médias numériques et de la culture numérique en prenant le cas de la société vietnamienne. Ses recherches portent sur l'identité collective, l'activisme en ligne, les imaginaires sociaux, la diaspora vietnamienne, les pratiques de foi catholique et la privacy.

Résumé : Cette étude entend offrir un éclairage sur le rapport entre la foi catholique et les dispositifs numériques dans la situation particulière des catholiques vietnamiens diasporiques. Sur le plan épistémologique, l'approche communicationnelle mettra en évidence l'articulation entre ce qu'offrent les dispositifs numériques et ce qu'en font des usagers. À travers une recherche documentaire sur la constitution de la population catholique vietnamienne d'outre-mer, l'analyse sémiotique de deux sites web et une enquête sociologique par questionnaires et entretiens, ce travail mettra en lumière l'appropriation des médias numériques par cette population et sa façon propre d'imaginer de nouvelles manières de pratiquer sa foi en situation de dispersion. L'enquête montrera une catholicité vietnamienne d'outre-mer en dynamique imaginative qui soulève le défi de repenser la compréhension de l'Église catholique en général sous l'angle du rapport entre le « en 


\section{Anh-Ngoc HOANG}

ligne » et le « hors ligne », entre l'ancrage solide des traditions et les connections « liquides » contemporaines.

Mots-clés: Catholiques, diaspora vietnamienne, médias numériques, pratiques religieuses en ligne.

Abstract: Catholic Faith and Digital Media. The case of the Vietnamese Diaspora - This study aims to provide initial insights into the relationship between the Catholic faith and digital media through the particular case of the Vietnamese Catholic diaspora. Drawing on an "epistemology of complexity", this investigation attempts to articulate new possibilities offered by sociotechnical objects with actual usages and practices by Internet users. Through a semiotic analysis of two websites and a sociological survey by questionnaire and interviews, the study will show the way this diasporic population uses digital devices to imagine new practices of their Catholic faith in a situation of geographic dispersion. It highlights a particular Catholicism of overseas Vietnamese characterized by a transnational dimension and an integration of online and offline faith practices. This novel reality poses challenges as well as opening up new perspectives for the Roman Catholic Church in the digital age, now confronted with tensions between long-standing "solid" traditions and contemporary "liquid" connections.

Keywords: Catholics, Vietnamese Diaspora, digital media, faith practices online.

Resumen: La Fe Católica y los dispositivos digitales. El caso de la diáspora católica vietnamita - Este estudio quiere mostrar la relación entre la fe católica y los dispositivos digitales en una situación particular, como es la de los católicos vietnamitas de la diáspora. Sobre el plano epistemológico, el estudio comunicacional que cosntruye una "epistemología de la complejidad" pone en evidencia la articulación entre lo que ofrecen

tic\&société - 9 (1-2), 2015 
La foi catholique et les dispositifs numériques :

le cas de la diaspora catholique vietnamienne

los dispositivos digitales y lo que hacen los usuarios. A través del análisis semiótico de dos sitios web y de una encuesta sociológica mediante un cuestionario y de entrevistas-, este trabajo muestra la apropiación de los medios de comunicación digitales que realiza esta población; a la vez muestra también su forma propia de imaginar nuevas maneras de practicar su fe en situación de dispersión. De la encuesta se deduce una catolicidad vietnamita de ultramar en clave imaginativa que suscita el desafío de repensar la comprensión de la Iglesia católica en general desde el punto de vista de la relación entre el online y el offline, entre la solidez de las tradiciones y las conexiones contemporáneas "líquidas".

Palabras clave: Católicos, diáspora vietnamita, medios de comunicación digitales, prácticas religiosas en línea.

L'intérêt scientifique de ce travail de recherche portant sur les médias numériques des Vietnamiens catholiques est justifié par deux raisons. D'une part, il est généralement admis que transmission et communication sont au cœur de la mission des Églises et de la vocation des religions (Marechal, Méadel et Veyrat-Masson, 2011, p.6). Ainsi, la question de la communication en régime chrétien catholique présente un intérêt scientifique évident, car "le christianisme catholique peut apparaître comme un système total de communication » et " la culture chrétienne a peut-être constitué [...] une matrice de la communication oçcidentale moderne" (Douyère, 2010, p.17). De son côté, l'Église catholique n'a cessé d'exprimer son intérêt tout particulier pour les moyens médiatiques, abordés par le décret du concile Vatican II Inter Mirifica, en 1963, comme figurant "parmi les merveilleuses découvertes techniques qu'avec l'aide de Dieu, le génie de l'homme a tirées de la création » $\left(\S n^{\circ} 1\right)$. Cette même 


\section{Anh-Ngoc HOANG}

vision positive de l'Église catholique, concrétisée dans l'instauration de la "Journée mondiale des communications sociales ", se poursuit jusqu'à l'époque d'internet avec ces premiers mots exprimés dans un autre texte officiel de 2002 intitulé L'Église et Internet: "L'intérêt de l'Église pour Internet est une expression particulière de son intérêt de longue date pour les moyens de communication sociale » $\left(\S n^{\circ} 1\right)$.

D'autre part, force est de constater qu'il existe déjà des travaux traitant de la question de la religion catholique des Vietnamiens d'outre-mer (notamment Phan, 2000, pour les catholiques vietnamiens aux États-Unis ; Tran Van Toan, 1998, pour ceux de France ; Dorais, 2011, 2007, pour ceux du Canada ; Kawakami, 2003, pour ceux du Japon et d'Australie), mais plus rares sont ceux qui se penchent sur l'étude des médias catholiques vietnamiens, qui sont souvent appréhendés sous un angle essentiellement théologique et pastoral chrétien (voir Nguyen Ngoc Son, 2006 ; Le Dinh Bang, 2009 ; Ngoc Lan, 2010). Cependant, le champ spécifique concernant les rapports entre la population des catholiques vietnamiens en diaspora et les technologies de l'information et de la communication (Tic) reste, à notre connaissance, inexploité (à l'exception de l'article de Hüwelmeier, 2014). Ce travail se propose donc d'explorer ce champ encore largement en friche.

Dans cette perspective, cet article a pour objectif de proposer une première analyse de la façon dont les catholiques vietnamiens d'outre-mer mobilisent et s'approprient des dispositifs numériques dans la perspective qui leur est propre de tenter d'imaginer de nouveaux modes de pratique de leur foi catholique dans un mode de vie contemporain marqué par la dispersion géographique et l'omniprésence technologique. Ces nouvelles pratiques de foi catholique vietnamienne qui tentent d'intégrer le " en ligne » et le " hors ligne » montreront l'imaginaire d'un catholicisme vietnamien trans-nationalisé et conduisent à repenser la compréhension de l'Église catholique à l'ère du numérique.

tic\&société - 9 (1-2), 2015 
La foi catholique et les dispositifs numériques:

le cas de la diaspora catholique vietnamienne

L'article comportera trois parties. Dans un premier temps, sur le plan épistémologique, le choix d'une approche communicationnelle a pour objectif de saisir la complexité des dispositifs numériques en tant qu'objets en cours de stabilisation et il justifiera le recours à une triple approche méthodologique, à savoir une recherche documentaire sur la constitution de la population catholique vietnamienne d'outre-mer, une analyse sémiotique des dispositifs et une enquête sociologique par questionnaires et entretiens auprès des usagers catholiques vietnamiens. Dans un deuxième temps, une présentation succincte sera consacrée à la constitution de la population de catholiques vietnamiens diasporiques dans le contexte des mouvements migratoires vietnamiens à l'époque contemporaine. La troisième et dernière partie portera sur l'analyse de deux premiers résultats de ce travail de recherche.

\section{Approche communicationnelle : une épistémologie pour saisir la complexité des dispositifs numériques}

\subsection{Un cadre théorique au croisement d'approches}

Dans la lignée de certains travaux menés en sciences de l'information et de la communication (Le Marec, 2002 ; Davallon, 2004 ; Monnoyer-Smith, 2008), ce travail s'inscrit dans une posture communicationnelle conçue comme "une épistémologie de la complexité ", à savoir qu'elle construit des objets de recherche "sur la complexité et l'hétérogénéité intrinsèque des objets sociotechniques autour et à travers lesquelles se constituent les pratiques sociales analysées » (Monnoyer-Smith, 2008). Prenant en compte "l'inscription de la matérialité des supports de communication dans les pratiques sociales 》 (Ibid.), c'est-à-dire s'attachant «à des complexes et non à des objets unitaires " (Davallon, 2004, p.34) ou à des « composites » (Le Marec, 2002), 


\section{Anh-Ngoc HOANG}

le regard communicationnel peut offrir un éclairage particulier par " son aptitude à articuler [...] des processus et des techniques, des pratiques et des dispositifs 》 (Monnoyer-Smith, op.cit.).

Par ailleurs, elle se nourrit également de la théorie de la "médiation de sens » telle qu'elle est proposée par Stewart Hoover soit la recherche des significations sociales que les médias ont pour les gens, et l'accent mis sur le côté de réception de la communication (Hoover, 2006, cité par Lundby, 2013, p.230). Aussi dans le sillage des travaux de Nick Couldry qui vont au-delà des médias conçus seulement comme institutions, textes ou objets technologiques, cette étude considère des médias sous l'angle des pratiques ; cela signifie se demander ce que les gens font dans le cadre de médias à travers toute une gamme de situations et de contextes (Couldry 2004, cité par Lundby, 2013, p.230).

Et puis, ayant affaire à des usages de dispositifs numériques, c'est-à-dire dans un cadre où "les phénomènes observés sont encore en cours de stabilisation» (Coutant et Domenget, 2014, p.231), cette étude s'appuiera sur une topique élaborée par la sociologie critique des usages et composée de quatre catégories analytiques: l'usage (ce que les gens font effectivement avec le dispositif), la pratique (l'activité quotidienne plus large de l'individu ou du groupe qui repose sur l'usage de différents dispositifs), les représentations mentales et sociales de la technique, et le contexte, social, politique et culturel (Jauréguiberry et Proulx, 2011, cités par Coutant et Domenget, 2014, p.235).

Enfin, cette recherche se trouve aussi enrichie de réflexions théoriques portant sur les rapports complexes qu'entretiennent des populations contemporaines en mouvement, baptisées désormais "diasporas ", avec les technologies de l'information et de la

${ }^{1}$ Nous proposerons plus loin une discussion du terme « diaspora » et notre justification quant à son utilisation pour le cas des catholiques vietnamiens en dispersion.

tic\&société - 9 (1-2), 2015 
La foi catholique et les dispositifs numériques:

le cas de la diaspora catholique vietnamienne

communication depuis la seconde moitié des années $1990^{2}$. Ainsi, en nous appuyant sur les travaux d'Arjun Appadurai (1996), nous souscrivons à la thèse d'une imagination collective en tant que pratique sociale à l'œuvre à l'ère de la globalisation où les médias et la migration sont deux facteurs majeurs qui rendent possible la nouvelle force de cette imagination collective.

\subsection{Des méthodologies plurielles}

Une fois posée, cette posture épistémologique permet d'adopter une démarche méthodologique plurielle, susceptible de tenir compte de cette complexité du phénomène observé.

D'abord, une recherche documentaire vise à saisir le contexte de la naissance de ces communautés diasporiques des catholiques vietnamiens en la situant dans le mouvement général de migrations importantes des Vietnamiens au cours du XXe siècle. Cette analyse permettra de discuter dans quelle mesure l'usage de l'expression « diaspora catholique vietnamienne » pourra faire sens.

Puis, une approche sémiologique portera sur deux sites web significatifs de la diaspora catholique vietnamienne, à savoir Vietcatholic.net et Mekhiettam.org, choisis sur la base de leur popularité, de leur ancienneté ${ }^{3}$ ou de leur signification ${ }^{4}$.

2 Tristan Mattelart a proposé une lecture critique de l'émergence de ce champ de recherche dans son article "Les diasporas à l'heure des technologies de l'information et de la communication : petit état des savoirs ", publié dans cette revue, tic \& société, en 2009 (voir : Mattelart, 2009 b).

3 Vietcatholic.net a été classé premier dans la liste des dix (Top Ten) sites web catholiques vietnamiens les plus populaires par le classement d'Alexa.com en 2013 ; voir <http://www.chuacuuthe.com/2013/03/alexa-10-website-cong-giao-viet-nam/> (dernière consultation le 5 novembre 2014). Par ailleurs, se présentant comme ayant été créé en 1996, Vietcatholic.net figure parmi les premiers sites catholiques vietnamiens dans le monde. 


\section{Anh-Ngoc HOANG}

Enfin, une enquête sociologique sur les usages et les représentations des dispositifs numériques sera mise en œuvre auprès des usagers catholiques vietnamiens au Việt Nam et d'outre-mer, sous une double forme : un questionnaire puis un entretien semi-directif.

L'enquête s'est, en effet, déroulée en deux étapes:

- première étape : un questionnaire exploratoire composé principalement de questions ouvertes, mené de janvier à mars 2014, qui a donné lieu à une trentaine de réponses de la part des catholiques vietnamiens du pays et d'outre-mer ;

- seconde étape : un nouveau questionnaire, composé principalement de questions fermées, formulées sur la base des réponses de la première étape de l'enquête, a été effectué d'août à octobre 2014, avec près de 132 réponses des vietnamiens catholiques nationaux et 46 réponses des vietnamiens catholiques diasporiques. En outre, un entretien a été mené auprès d'une dizaine de personnes catholiques vietnamiennes du Vietnam et d'ailleurs.

Dans la première étape, les questionnaires ont été diffusés, sous forme de document Word, via messagerie personnelle, dans un milieu catholique proche du chercheur en raison de la phase exploratoire de cette étude, en recherchant la diversité sur le plan international et en termes d'états de vie des répondants. Mon choix s'est orienté vers des catholiques vietnamiens ou d'origine

\footnotetext{
4 Vietcatholic.net se donne à voir, par son nom même, comme un site web de et pour l'ensemble de la catholicité vietnamienne. Mekhiettam.org (dont le nom signifie "Notre Dame du Cœur pur ») est un site web qui entend s'adresser aux Vietnamiens catholiques dans le monde et qui gère un salon de discussion publique (chat room) sous le logiciel Paltalk, nommé "Mẹ Khiết Tâm Bảo Vệ Sự Sống" ["Notre-Dame du Cœur pur, Protectrice de la vie"], dédié à la prière en ligne et à des activités caritatives auprès des pauvres et des malheureux au Vietnam.
}

tic\&société - 9 (1-2), 2015 
La foi catholique et les dispositifs numériques:

le cas de la diaspora catholique vietnamienne

vietnamienne, pratiquants, en milieu urbain, et plutôt de classe moyenne.

Dans la seconde étape, à partir du premier réseau d'enquêtés, j'ai tenté d'élargir le cercle des enquêtés en proposant aux premiers de faire passer le nouveau questionnaire à leur entourage (famille, paroissiens, amis catholiques, membres d'un même mouvement ou d'une même association religieuse, etc.). Le questionnaire était mis en ligne à travers le service Google Drive.

\section{Les Vietnamiens catholiques d'outre-mer: grands traits d'une communauté diasporique}

II semble évident que l'établissement des communautés vietnamiennes catholiques hors du Việt Nam s'inscrit dans l'histoire générale des mouvements migratoires vietnamiens, notamment durant le siècle dernier. Sans entrer dans les détails de ce phénomène complexe (pour une plus ample présentation de I'histoire de l'émigration vietnamienne au $\mathrm{XX}^{\mathrm{e}}$ siècle, voir Hoang et Vigne, 2014), on estime, en 2012, à environ quatre millions le nombre de Vietnamiens à l'étranger (descendants inclus), notamment aux Etats-Unis (1, 5 million), en France (300 000), au Canada (250 000), en Australie (245 000), en Allemagne (100 000) selon la Review of Vietnamese Migration Abroad (2012, p.29). C'est au sein de cette population hétérogène (périodes et motivations de départ, profils de migrants, lieux de réinstallation, etc.) que se sont établies des communautés catholiques vietnamiennes diasporiques.

Au sujet du mouvement migratoire des Vietnamiens catholiques à l'époque contemporaine, il faudrait mentionner au préalable l'exode massif interne des catholiques nord-vietnamiens en 1954, connu sous le nom du mouvement «Bắc di $c u$ ». Estimés à un million de personnes, ces migrants internes nord-vietnamiens, 


\section{Anh-Ngoc HOANG}

composés essentiellement de catholiques, se sont réfugiés dans le Sud-Việt Nam pour des raisons religieuses, mais aussi socioculturelles, économiques et politiques.

Puis, suite à la prise de Saigon en avril 1975 et à l'arrivée du régime communiste dans l'ensemble du Việt Nam, l'Église catholique vietnamienne a vécu un temps de grande répression et de persécution : confiscation du grand nombre des propriétés de l'Église dans le Sud-Việt Nam (établissements scolaires, établissements religieux, hôpitaux, orphelinats, imprimeries, etc.), expulsion de tous les missionnaires et du nonce apostolique, emprisonnement de l'évêque auxiliaire de Saigon, fermeture de tous les séminaires de 1975 à 1986, incarcération de prêtres dans des " camps de rééducation » où ils se trouvent emprisonnés avec des intellectuels, des hommes politiques, des soldats et tous ceux qui avaient collaboré avec le régime sud-vietnamien, etc.

Ainsi, aggravée par d'autres bouleversements socioéconomiques et politiques de l'après-1975, cette situation de répression religieuse a poussé de nombreux catholiques vietnamiens, parmi plus d'un million de Vietnamiens, à quitter leur terre natale pour trouver refuge et sécurité outre-mer.

En 2003, on dénombrait, sur une population estimée à 2 millions de Vietnamiens d'outre-mer, environ entre 500000 et 550000 catholiques, répartis sur 37 pays différents sur les cinq continents dont 200000 aux Etats-Unis, 75000 en France, 35000 en Australie, 20000 au Canada, 10000 en Allemagne, 700 en Italie. Parmi eux, il y avait 1036 prêtres, 165 religieux et des milliers de religieuses qui étaient au service de plus de 300 communautés catholiques diasporiques dans le monde (statistiques fournies par Đỗ Hữu Nghiêm A., Nguyễn Ngọc Sơn A. Trần Qúy Thiện J., 2004, p.874 et par l'agence Fides, 2003). Aujourd'hui, faute de statistiques récentes, mais en s'appuyant à la fois sur le chiffre précité de l'agence Fides et d'autres sources (Phan, 2000), on pourrait estimer à un tiers ou un quart de la population vietnamienne d'outre-mer totale le nombre des Vietnamiens catholiques, soit entre 1 million et 1,3 million.

tic\&société - 9 (1-2), 2015 
La foi catholique et les dispositifs numériques:

le cas de la diaspora catholique vietnamienne

Se pose maintenant la question de savoir comment nommer ces populations vietnamiennes spécifiques? En nous appuyant sur les travaux de Stéphane Dufoix (2011), l'usage de l'expression "diaspora catholique vietnamienne » pourra faire sens, à différents niveaux et selon différentes acceptions. Sur le plan étymologique, "Diaspora est un mot grec, fabriqué à partir du verbe grec diaspeírô, et signifiant 'dispersion', 'éparpillement'» (Dufoix, 2011, p.45). La dispersion géographique de fait de cette population catholique vietnamienne justifie donc l'usage " diaspora catholique vietnamienne " au sens étymologique. Sur le plan historique, diaspora est porteuse d'une "charge négative lourde de malheur, de persécution et de punition, essentiellement liée à l'histoire du peuple juif » (ibid., p.31). L'exode des catholiques vietnamiens en raison des mesures de répression et de persécution dont ils étaient victimes à partir de 1975 permet de les considérer comme «diasporiques". Sur le plan théologique chrétien, par ailleurs, dans le Nouveau Testament "diaspora " "correspond à une formule ecclésiologique désignant les membres de l'Église du Christ dont la condition terrestre est celle de l'étranger de passage » (Arowele, Diaspora-Concept in the New Testament, 1977, cité par Dufoix, 2011, p.79.). Ainsi, les catholiques vietnamiens hors du Việt Nam, suivant cette conception théologique de la diaspora, vivraient finalement une condition diasporique, comme tout chrétien sur terre, tenu " en dehors de sa vraie patrie qui est le royaume des cieux, la Jérusalem céleste » (ibid., p.80).

À la lumière de ces éléments concernant leur émergence dans I'histoire, les catholiques vietnamiens d'outre-mer ont formé une communauté diasporique en référence à la diaspora juive, c'est-àdire une dispersion caractérisée essentiellement par un événement douloureux, une mémoire collective, un désir et le fait de maintenir des relations avec le pays d'origine et l'Église d'origine, et l'existence de réseaux transnationaux de communautés catholiques vietnamiennes. 


\title{
Anh-Ngoc HOANG
}

\begin{abstract}
À titre d'illustration, on peut noter l'existence de deux organisations de nature transnationale rassemblant des catholiques vietnamiens du monde: d'une part, le Bureau de coordination pour la pastorale des Vietnamiens de la diaspora, une instance vaticane, créée en 1986 par la Congrégation pour l'évangélisation des peuples, basée à Rome; d'autre part, le Mouvement des laïcs vietnamiens de la diaspora, une association de fidèles laïcs créée en 1992, placée sous la tutelle du Conseil pontifical pour les laïcs et forte d'un réseau transnational de 42 bureaux basés aux Etats-Unis, en France, en Allemagne, en Australie, etc. (Đỗ Hữu Nghiêm, Nguyễn Ngọc Sơn A. , Trần Qúy Thiện, 2004, pp.975-880). Force est de constater l'usage explicite du terme de « diaspora » par ces instances officielles catholiques.
\end{abstract}

\section{L'imaginaire d'une catholicité vietnamienne en situation diasporique à l'ère du numérique}

\subsection{Des connexions numériques à la communion ecclésiale : transnationalisation d'un catholicisme vietnamien}

Parmi les trois catégories d'activités religieuses principales en ligne, à savoir celles liées aux "préoccupations spirituelles ou religieuses personnelles", celles liées à "la religion institutionnelle traditionnelle ", et celles concernant " la recherche de nouvelles en ligne " (Hoover, Schofield Clark et Rainie, 2004, cité par Arasa, Cantoni et Ruiz, 2010, p.33), il est intéressant de noter que l'un des services importants que proposent les sites web des catholiques vietnamiens d'outremer est de donner des nouvelles en ligne sur des événements et des affaires catholiques au Việt Nam ainsi que dans les communautés catholiques d'outremer et dans le monde. Cette dimension informationnelle maintient les visiteurs "connectés " aux diverses réalités des catholiques vietnamiens et de l'Eglise catholique, où qu'ils soient.

tic\&société - 9 (1-2), 2015 
La foi catholique et les dispositifs numériques:

le cas de la diaspora catholique vietnamienne

Le site Vietcatholics.net en est une illustration significative. En fait, la section intitulée «Nouvelles de l'Église vietnamienne » [Tin Giáo Hội Việt Nam] comporte sans distinction des événements et des affaires catholiques à la fois au Việt Nam et dans les communautés catholiques vietnamiennes d'outremer (fig.1). Voici quelques titres d'articles de la première partie de cette section du 5 novembre 2014 : au Viêt Nam : "Célébration de la messe pour les fidèles défunts au cimetière des fœetus humains Dong Tien- Lagi », "La paroisse Chu Hai, Ba Ria: le mois de prières pour les âmes "; dans les communautés d'outre-mer : "Commémoration de l'ancien président Ngo Dinh Diem aux Pays-Bas », "La communauté catholique vietnamienne à San Jose visite un cimetière ", "Paris commémore les responsables militaires de la République du Việt Nam à Nogent sur Marne ». 


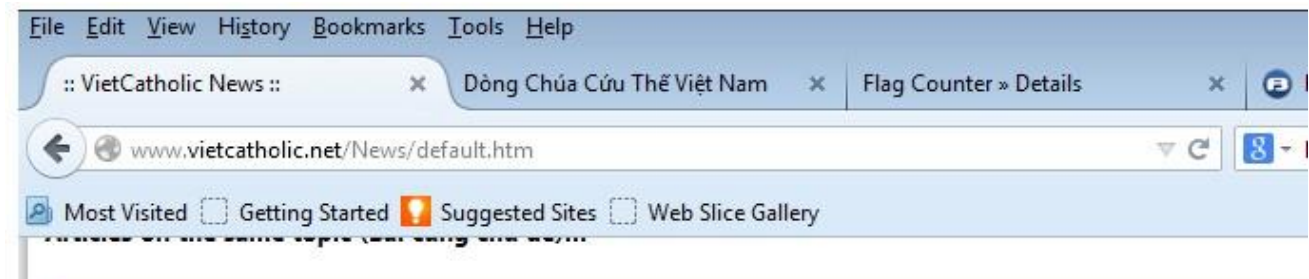

\section{Tin Giáo Hội Việt Nam}

Lễ Cầu cho Các Tín Hữu Đã Qua Đời tại Nghĩa Trang Thai Nhi Đồng Tiến - Lagi

Lm Giuse Nguyễn Hữu An

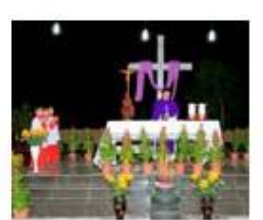

Nhin lá vàng rơi, ta nhớ lời Thánh Vinh: "Đời sống con người giỡng như cây cỏ, như bông hoa nở trên cánh đờng, một cơn gió thoảng đủ làm nó biến đi, nơi nó mọc không còn mang vêt..

> Cộng Đồng Công Giáo Việt Nam San Jose viếng nghiã trang Têrêsa Đinh

> Paris Tưởng Niệm các Chiến Sĩ Quân Lực VNCH tại Nogent Sur Marne TV Paris 13

L Lễ giỗ cố TT Ngô Đình Diệm tại Hòa Lan Thanh Sơn
Lễ Tưởng Niệm 51 Năm Cố Tổng Thống Ngô Đình Diệm và Quân Dân Chính VNCH tại Frankfurt Trungduong Tnguyen

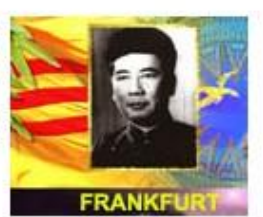

Frankfurt, Đức Quốc: Lễ Tường Niềm 51 Năm Cố Tổng Thống Ngô Đình Diệm, Cỗ Vã̃n Ngô Đình Nhu và những Quân Dân Chính Việt Nam Cộng Hòa đã Vị Quốc Vong Thân do nhóm anh em tại...

v Hình ảnh Lễ Giỗ 51 năm Cố TT Ngô Đình Diệm tại GX Ngôi Lời Nhập Thể, Houston Joseph Nugyễn Ký

- Giáo xứ Chu Hải, Bà Rịa: Tháng cầu cho các Linh hồn Đặng Hoàng Phúc

- Văn nghệ gây quỹ từ thiện của hội việt-Úc Bác ái Hội

Fig.1 : Capture d'écran du site Vietcatholic.net le 5 novembre 2014.

Ainsi, ce site considère tous ces événements, qu'ils se produisent au Việt Nam ou ailleurs (États-Unis, France, Australie, Allemagne, Pays-Bas, etc.), comme appartenant à une entité ecclésiale commune, à savoir "l'Eglise catholique vietnamienne ". Cette identification intentionnelle des différentes réalités par ce média numérique reflète autant qu'elle construit une représentation spécifique selon laquelle les communautés catholiques vietnamiennes de la diaspora font toujours partie de l'Église catholique vietnamienne.

La même vision est perçue sur la page d'accueil du site web Mekhiettam.org. On peut remarquer la présence de cinq horloges qui donnent des indications horaires dans cinq villes différentes: Californie, New-York, Saigon, Paris et Queensland (fig.2a). En fait, ce site suggère aux internautes de rejoindre son salon de prière en

tic\&société - 9 (1-2), 2015 
La foi catholique et les dispositifs numériques:

le cas de la diaspora catholique vietnamienne

ligne, basé sur Paltalk, à quatre horaires différents chaque jour : 3 heures, 8 heures, 14 heures et 20 heures (fig.2b) ${ }^{5}$.

Selon $\mathrm{M}^{\mathrm{me}}$ Lan - la fondatrice de ce média numérique (un salon de discussion et un site web) - qui vit au Danemark, ces quatre horaires sont mis en place de cette manière afin que les catholiques vietnamiens dans différents pays et continents (Việt Nam, Europe, USA et Australie) puissent rejoindre un créneau de prière en ligne. Donc, ce site vise à atteindre, par son fonctionnement, des visiteurs catholiques vietnamiens dans le monde entier. En reliant ces catholiques vietnamiens à travers le monde dans un culte partagé en ligne et dans d'autres activités communes que nous verrons plus loin, ce média numérique permet de transnationaliser le catholicisme vietnamien d'aujourd'hui.

5 Me Khiêt Tâm, <http://mekhiettam.org/home/?a=about>, dernière consultation le 5 novembre 2014. 


\section{Anh-Ngoc HOANG}

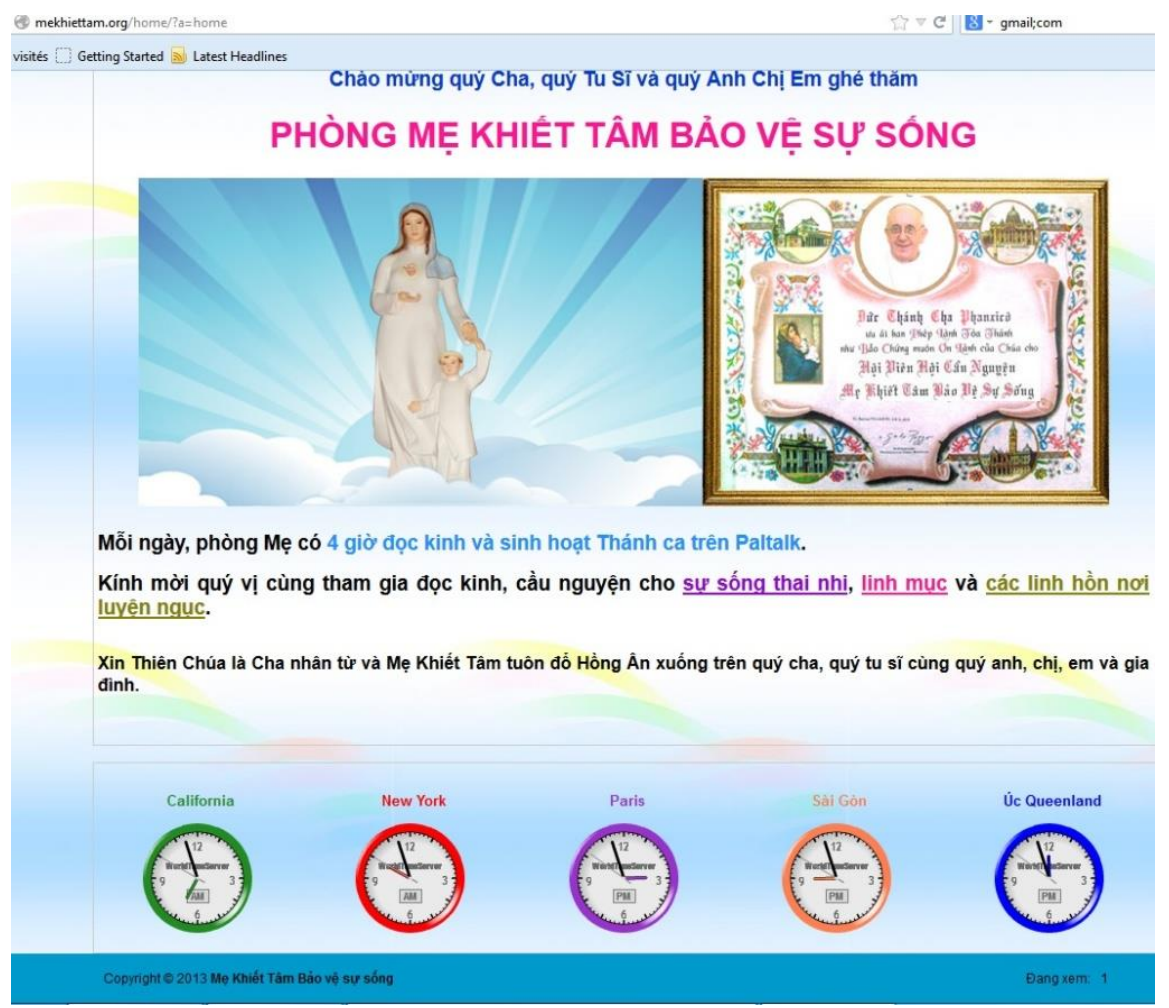

Fig.2a : Capture d'écran de la page d'accueil du site Mekhiettam.org du 5 novembre 2014.

tic\&société - 9 (1-2), 2015 
La foi catholique et les dispositifs numériques :

le cas de la diaspora catholique vietnamienne

\section{Giỡ Câu Nguyện mỗi ngày trên Paltalk}

Cầu cho sự sống của Thai Nhi, Linh Mục và các Linh Hồn nơi Luyện Ngục

- 03.00 giờ Việt Nam: 15 Kinh Nguyện Đặc biệt Cầu Cho Các Linh Hồn

- 08.00 giỡ Việt Nam: Chuối lệ

- 14.00 giỡ Việt Nam: Lân Hạt Lòng Thương Xót Đặc biệt Cầu Xin Chữa Lành

- 20.00 giỡ Việt Nam: Lân Hật Mân Côi Câu Cho Thai Nhi và Các Bà Mẹ

Fig.2b : capture d'écran du site Mekhiettam.org,

Indiquant les horaires de prière en ligne, le 5 novembre 2014.

En outre, ces médias numériques catholiques vietnamiens informent aussi sur des événements et des affaires catholiques dans le monde, en particulier ceux concernant le Vatican et le pape. Par conséquent, ils créent pour ces utilisateurs catholiques vietnamiens un sentiment d'être en «liens numériques » avec d'autres croyants catholiques et avec le Vatican, en d'autres termes avec ce qu'on appelle, selon la terminologie catholique, «l'Église universelle ». Par exemple, la section « Nouvelles de l'Église universelle » (Tin Giáo Hội Hoàn Vu) du site Vietcatholic du 18 novembre 2014 donne à lire un compte-rendu des diverses activités du pape François, de la fête de Thanksgiving aux ÉtatsUnis, de la situation actuelle des chrétiens en Turquie, etc. Par ailleurs pour produire ces nouvelles portant sur le Vatican et le monde catholique en langue vietnamienne, ces sites catholiques vietnamiens reproduisent principalement des informations à partir de sources officielles telles que Zenit.org, Catholic News Agency, Rome Reports, AsiaNews.it.

II importe maintenant d'examiner quels usages les internautes catholiques vietnamiens mettent en œuvre effectivement et quelles représentations ils se forgent des implications de ces connexions numériques sur leur foi.

En ce qui concerne les usages effectifs, les résultats obtenus grâce à notre enquête par questionnaires montrent que "s'informer sur l'Église catholique vietnamienne au Việt Nam " 


\section{Anh-Ngoc HOANG}

constitue ce qui intéresse la plupart des informateurs lors de leur visite des sites catholiques vietnamiens (57\% des informateurs de la diaspora l'ont choisi comme leur premier choix).

Quant aux représentations d'internautes, répondant à la question de l'utilité des médias numériques catholiques vietnamiens pour leurs pratiques religieuses, la majorité des informateurs catholiques vietnamiens diasporiques $(87 \%)$ ont été d'accord avec cette description : "Aider à mettre à jour, des nouvelles concernant sa paroisse, sa communauté, l'Église catholique vietnamienne au Việt Nam et d'outre-mer, l'Église catholique universelle. »

Cette représentation d'un impact positif des médias numériques catholiques vietnamiens sur les liens et les connexions entre les catholiques vietnamiens au Việt Nam et ceux d'outre-mer a été abondamment exprimée dans des réponses anonymes à une question ouverte de notre questionnaire ${ }^{6}$ dans les termes suivants : "Ils aident les chrétiens [vietnamiens] à être informés de la situation de l'Église locale et celle de l'Église universelle»; «lls permettent aux catholiques vietnamiens nationaux de voir que les catholiques vietnamiens d'outre-mer font également partie de l'Église catholique vietnamienne ».

Ainsi, nous constatons ici une cohérence entre les représentations et les usages des internautes concernant la dimension de mise en lien des médias numériques catholiques vietnamiens. Mais ce qui mérite une analyse plus approfondie pour saisir cet aspect apparemment «technocentriste » de la connectivité numérique est une signification spirituelle et religieuse que cette capacité de connexion pourrait véhiculer. En fait, les "connexions » rendues possibles par les médias numériques sont conçues et perçues par les catholiques vietnamiens dans la perspective de la "communion» chrétienne, une valeur

${ }^{6}$ La question posée était la suivante : «Selon vous, les médias numériques catholiques vietnamiens d'outre-mer contribuent-ils à l'Église catholique au Vietnam ? ».

tic\&société - 9 (1-2), 2015 
La foi catholique et les dispositifs numériques:

le cas de la diaspora catholique vietnamienne

fondamentale pour les croyants en général, sur le plan de l'anthropologie religieuse (Delaunay, 2014), et pour les catholiques en particulier.

D'un point de vue religieux, la «communion » se réfère à « une double dimension d'accomplissement spirituel de l'être humain, a) une dimension de communauté, impliquant les notions de partage, d'échange, de réunion, de témoignage, de service, de don, de convivialité ; $b$ ) une dimension d'union personnelle, impliquant les notions de présence, de participation, de fusion avec le divin, d'adoration, d'accomplissement, d'unicité » (Delaunay, 2014). D'un point de vue catholique, en particulier, la «communion » signifie " la communauté unie des fidèles ou des saints, ou de l'Eglise comme un corps de croyants. [...] La communion unit l'individu à la fois verticalement et horizontalement avec Dieu, avec son prochain dans une communion avec le Christ » (Flinn, 2007, p.171).

En effet, le terme "communion » [Hiệp thông] a été utilisé de nombreuses fois dans des réponses ouvertes de la part de nos informateurs, quand ils expriment leur perception des avantages des médias numériques vietnamiens catholiques : "Ils créent de la communion et des liens entre les catholiques vietnamiens au Việt Nam et ceux des communautés d'outre-mer »; " lls permettent à tous les chrétiens dans l'Église d'être en communion»; «lls permettent à l'Église au Việt Nam de voir l'unité et la communion de la famille catholique vietnamienne indépendamment du lieu ».

Cette perception a été également confirmée par la majorité des informateurs (87\%) exprimant leur accord sur la description suivante relative à l'utilité des médias numériques catholiques vietnamiens dans la vie catholique: "Aider à construire la communion entre des catholiques vietnamiens; entre l'Église vietnamienne et l'Église universelle ».

Loin d'être issue des caractéristiques technologiques de ces médias numériques, cette "communion " est conçue par ces catholiques vietnamiens comme un fruit de la prière, de la compréhension, de partage, de soutien et d'ouverture : "lls créent 


\section{Anh-Ngoc HOANG}

de la communion dans la prière et l'évangélisation »; «Les médias numériques des catholiques vietnamiens d'outre-mer apportent du partage, de l'ouverture et du soutien à l'Église catholique vietnamienne au Việt Nam »; (lls offrent) « Plus de compréhension au sujet de la vie de l'Église dans le monde "; "La lecture des informations et des témoignages des chrétiens de partout dans le monde permet de prendre conscience de son appartenance à l'Église, incite à prier pour des Églises en persécution ».

Ainsi, il a été démontré que les médias numériques catholiques vietnamiens ont rendu possibles et visibles des connexions et des liens entre les catholiques vietnamiens du pays et ceux de la diasspora, entre l'Église catholique romaine du Việt Nam et l'« Eglise universelle ». Ces liens numériques jouent un rôle significatif dans le développement d'un sens contemporain de la «communion ecclésiale» entre les diverses communautés catholiques vietnamiennes qui sont dispersées géographiquement dans le monde entier. Dans cette perspective, ils contribuent à façonner un catholicisme vietnamien transnationalisé contemporain.

Outre cette question de la «communion ecclésiale », la communication numérique par les catholiques vietnamiens d'outremer permet également d'imaginer de nouvelles pratiques religieuses à l'ère du numérique.

3.2. Au-delà des valeurs anciennes et des nouvelles pratiques: invention de nouvelles façons d'être catholique vietnamien dans un contexte des pratiques hybrides en ligne et hors ligne

3.2.1. Vers des pratiques de foi catholique de plus en plus soutenues par des technologies numériques

Le site diasporique Vietcatholic illustre bien cet aspect. Sur la partie droite de la page d'accueil du site du 8 décembre 2014, on

tic\&société - 9 (1-2), 2015 
La foi catholique et les dispositifs numériques: le cas de la diaspora catholique vietnamienne

peut cliquer sur la section intitulée «liturgie» pour afficher une liste d'articles qui représentent des méditations sur la Parole de Dieu, des homélies liées à la fête de l'Immaculée Conception, à la messe du $2^{\mathrm{e}}$ dimanche de l'Avent ${ }^{7}$, etc. (voir fig.3). À côté de cette section s'en trouve une autre appelée « liens » qui comporte, entre autres, les sous-sections telles que calendrier liturgique, homélies, prières de messe. Ici sont donnés plus de détails aux internautes catholiques vietnamiens pour les aider à pratiquer leur foi dans le domaine de la "liturgie " qui peut être compris simplement comme l'ensemble des actes officiels de culte de l'Église rendus à Dieu $^{8}$. Par exemple, le « calendrier liturgique » contient des dates, la couleur des vêtements à porter par le prêtre lors de chaque célébration, et les références de lectures de la Bible aux dates correspondantes (voir fig.4). Tous ces éléments représentent des normes très codifiées de l'Eglise catholique romaine concernant le service religieux pour assurer les pratiques communes à tous les croyants catholiques, où qu'ils soient dans le monde.

\footnotetext{
${ }^{7}$ L'avent est une période du temps liturgique catholique qui commence le $4^{\mathrm{e}}$ dimanche avant Noël. Pour une explication complète, on peut consulter le site du service national de la pastorale liturgique et sacramentelle (SNPLS) de l'Église catholique, consacré à la liturgie catholique <http://www.liturgiecatholique.fr/Avent.html?var recherche=avent $>$, dernière consultation le 5 novembre 2014.

8 Pour une explication théologique complète de cette notion, on peut se référer au «portail de la liturgie catholique » de la Conférence des évêques de France: $<$ http://www.liturgiecatholique.fr/Liturgie.html?artsuite $=0>$, dernière consultation le 5 novembre 2014.
} 


\section{Anh-Ngoc HOANG}

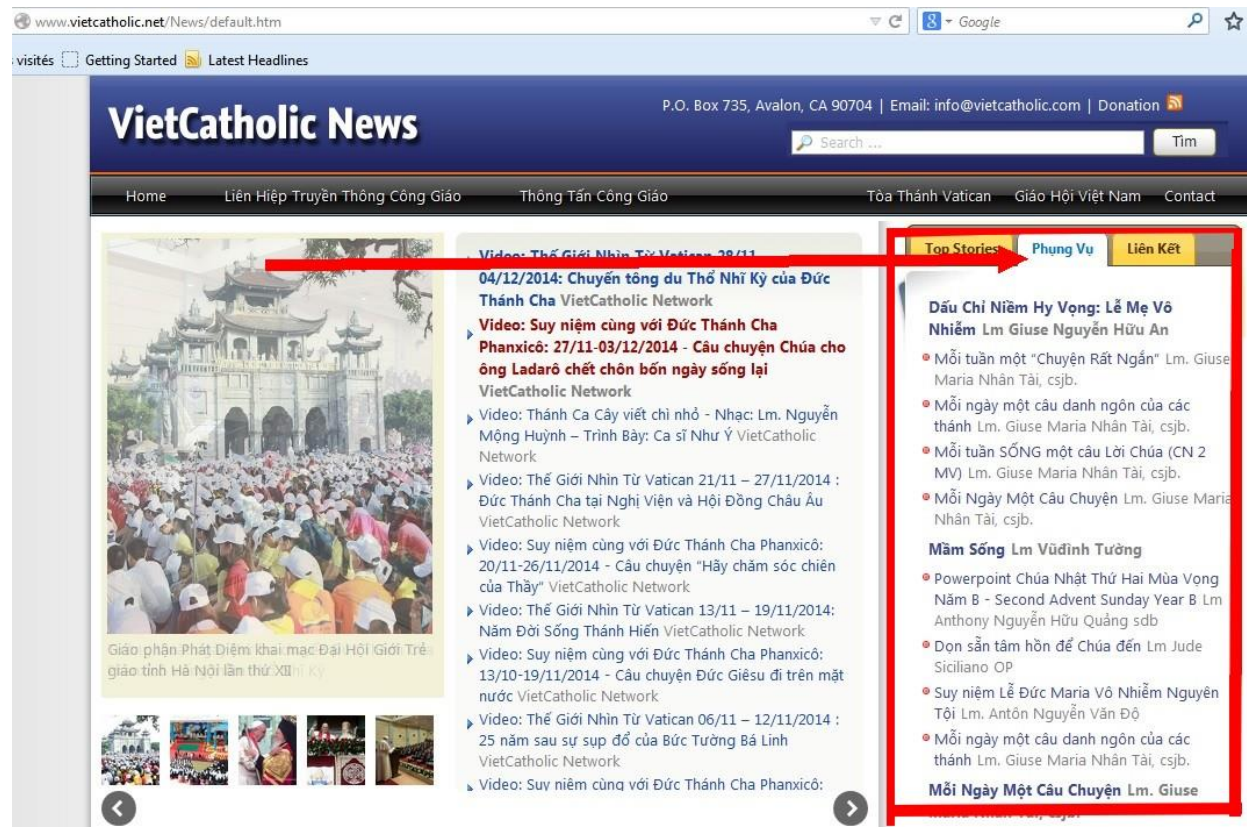

Fig.3 : Capture d'écran de la page d'accueil du site Vietcatholic du 8 décembre 2014 ; la section "Liturgie", à droite, est ici entourée d'un cadre rouge.

tic\&société - 9 (1-2), 2015 


\section{La foi catholique et les dispositifs numériques : le cas de la diaspora catholique vietnamienne}

us visités G Getting Started Latest Headlines

\section{Sich Phung Vu}

VietCatholic

\begin{tabular}{|c|c|c|}
\hline $\mathrm{DI}$ & $\dot{A}_{0} L^{2} e^{2}$ & Lễ \\
\hline $01 / 12$ & $\mathrm{Tm}$ & $\begin{array}{l}\text { Thứ Hai trong tuần thứ Nhất Mùa Vọng } \\
\text { Is } 2: 1-5 ; \text { Is } 4: 2-6 ; \text { Tv } 122: 1-2,3-4,4-5,6-7,8-9 ; \text { Mt } 8: 5-11\end{array}$ \\
\hline $02 / 12$ & $\mathrm{Tm}$ & $\begin{array}{l}\text { Thứ Ba trong tuàn thứ Nhất Mùa Vọng } \\
\text { Is 11:1-10; Tv 72:1,7-8,12-13,17; Lc 10:21-24 }\end{array}$ \\
\hline $03 / 12$ & $\mathrm{Tr}$ & $\begin{array}{l}\text { Thánh Phanxicô Xaviê, Lm } \\
\text { Is 25:6-10; Tv 23:1-3,3-4,5,6; Mt 15:29-37 }\end{array}$ \\
\hline $04 / 12$ & $\mathrm{Tm}$ & $\begin{array}{l}\text { Thứ Năm trong tuàn thư Nhất Mùa Vong } \\
\text { Is } 26: 1-6 ; \text { Tv 118:1,8-9,19-21,25-27; Mt 7:21,24-27 }\end{array}$ \\
\hline $05 / 12$ & $\mathrm{Tm}$ & $\begin{array}{l}\text { Thứ Sáu trong tuần thứ Nhất Mùa Vong } \\
\text { Is 29:17-24; Tv 27:1,4,13-14; Mt 9:27-31 }\end{array}$ \\
\hline $06 / 12$ & $\mathrm{Tm}$ & $\begin{array}{l}\text { Thứ Bấy trong tuần thứ Nhất Mùa Vong } \\
\text { Is 30:19-21,23-26; Tv 147:1-2,3-4,5-6; Mt 9:35; Mt 10:1,6-8 }\end{array}$ \\
\hline $07 / 12$ & $\mathrm{Tm}$ & $\begin{array}{l}\text { Chúa Nhật thứ Hai Mùa Vọng } \\
\text { Is 11:1-10; Tv 72:1-2,7-8,12-13,17; Rm 15:4-9; Mt 3:1-12 }\end{array}$ \\
\hline $08 / 12$ & $\mathrm{Tr}$ & $\begin{array}{l}\text { Lễ Đức Me Vô Whiễm Nguyên Tôi } \\
\text { Is 35:1-10; Iv 85:9-10,11-12,13-14; Lc 5:17-26 }\end{array}$ \\
\hline $09 / 12$ & $\mathrm{Tm}$ & $\begin{array}{l}\text { Thứ Ba trong tuần thứ Hai Mùa Vọng } \\
\text { Is 40:1-11; TV 96:1-2,3,10,11-12,13; Mt 18:12-14 }\end{array}$ \\
\hline $10 / 12$ & $\mathrm{Tm}$ & $\begin{array}{l}\text { Thứ Tư trong tuần thứ Hai Mùa Vong } \\
\text { Is } 40: 25-31 ; \text { Tv 103:1-2,3-4,8,10; Mt 11:28-30 }\end{array}$ \\
\hline $11 / 12$ & $\mathrm{Tm}$ & $\begin{array}{l}\text { Thứ Năm trong tuằn thứ Hai Mùa Vong } \\
\text { Is 41:13-20; Tv 145:1,9,10-11,12-13; Mt 11:11-15 }\end{array}$ \\
\hline $12 / 12$ & $\mathrm{Tr}$ & $\begin{array}{l}\text { Lể Đức Mẹ Guadalupe } \\
\text { Is 48:17-19; Tv 1:1-2,3,4,6; Mt 11:16-19 }\end{array}$ \\
\hline $13 / 12$ & $\bigoplus \dot{0}$ & Thảnh Lucia, Đttd \\
\hline
\end{tabular}

Fig. 4 : Capture d'écran de la sous-section intitulée « calendrier liturgique » du site Vietcatholic, le 8 décembre 2014. 


\section{Anh-Ngoc HOANG}

Dans la même perspective, il est intéressant d'examiner l'exemple du fichier Powerpoint téléchargeable appelé "Evangile du $2^{\mathrm{e}}$ dimanche de l'Avent 2014 » (fig.5). Ce document est un texte illustré et bilingue en vietnamien et en anglais d'un passage de la Bible, extrait du Nouveau Testament, et correspondant à la lecture de la messe du $2^{\mathrm{e}}$ dimanche de l'avent. L'utilisation d'un texte bilingue, anglais et vietnamien, montre une prise de conscience de la nécessité de s'adresser à un public diversifié dans les communautés catholiques vietnamiennes d'outre-mer où les jeunes générations d'origine vietnamienne ne comprennent pas toujours la langue vietnamienne ${ }^{9}$.

${ }^{9}$ En effet, dans notre questionnaire, $80 \%$ des informateurs diasporiques ont été d'accord avec la description suivante au sujet des limites des médias numériques pour les catholiques vietnamiens : "Incapacité à accéder aux sites web catholiques vietnamiens pour des jeunes d'origine vietnamienne qui ont grandi hors du Vietnam et qui ne lisent pas le vietnamien ».

tic\&société - 9 (1-2), 2015 
La foi catholique et les dispositifs numériques :

le cas de la diaspora catholique vietnamienne

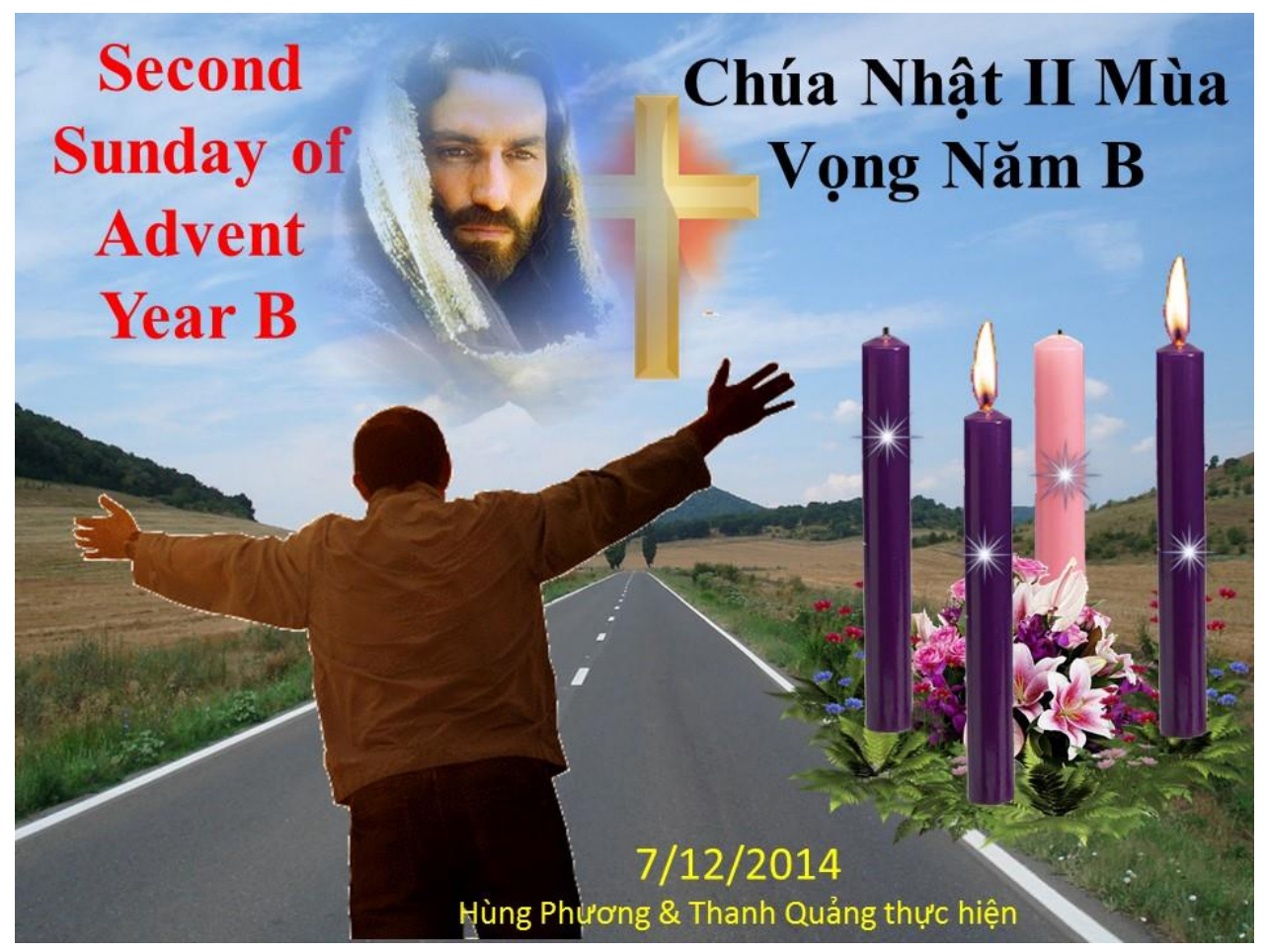

Fig.5 : Capture d'écran de la $1^{\text {ere }}$ page du document Powerpoint de l'évangile $d u 2^{e}$ dimanche de l'Avent sur le site Vietcatholic du 8 décembre 2014.

Le recours aux technologies numériques pour les pratiques de foi catholique est également intéressant sur le site Mekhiettam.org. Un exemple significatif est le programme de " retraite - formation spirituelle " annoncé sur le site que nous pouvons voir le 8 décembre 2014 (fig.6). La nouveauté réside ici dans l'offre d'une nouvelle pratique de foi, qui est la possibilité de suivre une retraite - formation à distance grâce à un support technologique qu'est un site web. En fait, pour chaque jour, les internautes peuvent 


\section{Anh-Ngoc HOANG}

librement télécharger les fichiers audio de la lecture biblique et de I'homélie qui ont été produits par le prédicateur en charge et affichés sur le site web à leur disposition. Sur la base des informations recueillies dans notre entretien avec la fondatrice de Mekhiettam.org, ce site est caractérisé par une dimension transnationale avec un directeur spirituel qui est un prêtre d'origine vietnamienne, basé en France, et une centaine de membres vietnamiens dispersés entre le Việt Nam, les États-Unis, l'Europe et l'Australie.

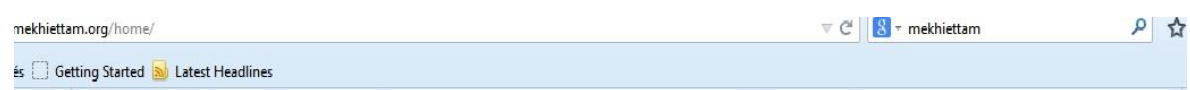

Tam Nhật kính lễ Mẹ Vô Nhiễm Nguyên Tội

Chương trình tĩnh huấn

Ngày 5-7 tháng 12 năm 2014

Lm Giuse Nguyễn Ngọc Dũng

Chủ đề : Đừng sợ mở cửa cho Chúa Thánh Linh.

Huyền nhiệm "Truyền Tin": Thiên Chúa gõ cửa lòng con người.

Thứ 5 ngày 4 tháng 12 Tải bài giảng Tải bài đọc

Lc 1,26-38: Tại sao Chúa Thánh Linh lại muốn gõ cửa lòng con người?

Thứ 6 ngày 5 tháng 12 Tải bài giảng Tải bài đọc

Lc1,26-38: Bằng cách thức nào, Đức Maria đã "mở lòng" ra cho Chúa Thánh Linh ?

Thư 7 ngày 6 tháng 12 Tải bài giảng Tải bài đọc

Lc1,26-38: Đâu là nguyên do của việc Đức Maria "mở lòng" ra với

tic\&société - 9 (1-2), 2015 
La foi catholique et les dispositifs numériques:

le cas de la diaspora catholique vietnamienne

Fig.6 - Capture d'écran du programme « Retraite - formation » sur le site Mekhiettam.org du 8 décembre 2014.

Ainsi, dans une tentative de répondre aux besoins religieux d'une population de catholiques vietnamiens géographiquement dispersés dans le monde qui ne peuvent pas toujours assister à une retraite-formation spirituelle "classique » organisée dans un centre spirituel local, ce site offre une nouvelle façon de vivre une expérience de la foi catholique. Cette nouvelle pratique correspondrait à la fois à un mode de vie de plus en plus mobile et à une foi plus personnalisée de nos contemporains.

Après avoir examiné quelques nouvelles possibilités offertes par ces médias numériques des catholiques vietnamiens, il est maintenant important de tenir compte des usages et des pratiques effectifs des internautes.

Tout d'abord, même s'ils ne passent pas beaucoup de temps en ligne (48\% des catholiques vietnamiens d'outre-mer ont déclaré passer de une à trois heures en ligne par jour), la majorité d'entre eux $(83 \%)$ considèrent l'internet comme une source principale d'informations et montrent des usages importants de médias numériques pour leur foi catholique, par exemple "regarder un programme catholique sur l'internet (sur Youtube, ou ailleurs) ", ou " lire un écrit catholique sur un site web ou un blog».

Ensuite, parmi les six centres d'intérêts les plus importants sur les sites catholiques vietnamiens, il est intéressant de noter la très grande importance gue les usagers diasporiques accordent au fait de s'informer sur l'Eglise catholique au Việt Nam, dans le monde et dans les communautés d'outre-mer (trois réponses sur six). Cela confirme notre analyse précédente quant à la construction de la «communion ecclésiale » que permettent désormais, entre autres, les supports médiatiques numériques catholiques.

Enfin, quant aux représentations des usagers catholiques vietnamiens, notre enquête montre que la plupart d'entre eux ont 


\section{Anh-Ngoc HOANG}

exprimé leur accord sur les nombreux avantages des médias numériques catholiques par rapport aux médias traditionnels (presse écrite, radio, télévision, etc.). Avec la même vision positive, ils considèrent les médias numériques catholiques vietnamiens très utiles pour leur vie de foi. Ainsi, les médias numériques catholiques vietnamiens sont perçus par les usagers comme des ressources documentaires, des outils de communion, un soutien à la prière et à la maturité intérieure, une aide à la formation religieuse.

En résumé, cette analyse a mis en lumière l'importance croissante des pratiques religieuses des catholiques vietnamiens à travers des médias numériques. Mais, comme il l'a été démontré, " les usagers d'internet connectent de manière conceptuelle et pratique leur vie sociale online et offline, plutôt que de les voir comme des sphères séparées ou déconnectées " (Wellman et Haythornwaite, 2002 ; Wellman 2004, cités l'un et l'autre par Campbell, 2013, p.63).

3.2.2. Vers une foi catholique combinant de façon croissante en ligne et hors ligne

Dans cette partie, une analyse détaillée sera effectuée des deux dispositifs numériques que forment le site Mekhiettam (<http://mekhiettam.org>) et le salon de discussion basé sur Paltalk nommé Me Khiet tam bao ve su song.

Force est de constater, d'emblée, la perspective de l'intégration de prière en ligne et d'actions hors ligne qui est explicitement envisagée dans la section "présentation ". En fait, "la vision" [Tôn Chi] détermine deux objectifs de ce salon de discussion: "Aimer Dieu et aimer les uns les autres », et explique : "Aimer Dieu signifie L'adorer n'importe où et n'importe quand. Le salon de discussion dispose de quatre horaires pour réciter des prières, faire des lectures bibliques et de la méditation, et chanter pour louer le Seigneur éternel. Aimer les uns les autres signifie prier pour sa famille, ses parents, les fœtus humains, les prêtres, et les

tic\&société - 9 (1-2), 2015 
La foi catholique et les dispositifs numériques:

le cas de la diaspora catholique vietnamienne

âmes du purgatoire. [Cela signifie également] apporter de l'amour aux orphelins, aux pauvres, aux personnes âgées et solitaires ${ }^{10}$.

Ainsi, initialement, ce dispositif propose un service de "culte » (au moins au sens partiel, non sacramentel) catholique en ligne qui comporte à la fois prière en ligne et des supports documentaires religieux prévus à cet effet. Dans cette perspective, ce cyberespace fonctionne vraiment comme un «lieu rituel », où le « rituel » pourrait être défini comme «l'engagement délibéré avec le sacré quel que soit le sacré pour ceux qui s'y impliquent" (Helland, 2013, p.27). À la suite de la distinction entre les « online rituels » qui sont des rituels effectués dans l'espace numérique, et les « rituels online " qui se réfèrent à des recommandations, des textes de rituels, et des documents sur les rituels qui peuvent être trouvés sur des sites web (Miczek, 2008 et Heidbrink, 2007, cités par Helland, 2013, p.36), on peut dire que le salon de discussion Me Khiet tam bao ve su song propose la " online prière ", tandis que le site web Me Khiet tam offre une "prière online " à ses usagers d'internet, par exemple les indications pratiques pour la récitation de la prière à la miséricorde divine (voir fig.7).

Ces indications permettent aux usagers de ce salon Paltalk de faire l'expérience d'un rituel de prière en ligne comme s'ils étaient dans un rassemblement de prière traditionnel présentiel. Plus profondément, les activités de foi catholique en ligne proposées dans ce dispositif numérique reflètent certaines dévotions populaires de longue date des catholiques vietnamiens, à savoir les dévotions mariales, la récitation de prières, les prières pour les âmes du purgatoire, et attestent également d'un grand respect visà-vis des prêtres dans le catholicisme vietnamien (Phan, 2000, p.24).

Pour résumer, le média numérique Me Khiet tam montre une tentative de rendre possibles certaines pratiques en ligne pour

${ }^{10}<$ http://mekhiettam.org/home/?a=about $>$, dernière consultation le 5 novembre 2014. 


\section{Anh-Ngoc HOANG}

« créer une version online d'une tradition de foi offline » (Campbell, 2013, p.61). Ces nouvelles formes de pratiques religieuses qui sont "technologiquement médiatées " ont émergé d'une communauté religieuse hybride qui cherche à intégrer des pratiques religieuses numériques et des expériences en coprésence, comme nous allons le voir maintenant.

tic\&société - 9 (1-2), 2015 


\section{La foi catholique et les dispositifs numériques : le cas de la diaspora catholique vietnamienne}

\section{LÒNG THƯƠNG XÓT CHÚA}

(Quý anh chị bấm vào mục màu HƠNG để hiển thị hoặc ẩn nội dung)

01. Lời Nguyện mờ đầu

02. Làm dấu Thánh Giá:

Nhân danh Cha † và Con † và Thánh Thần † Amen.

03. Hát hoặc Đọc Kinh Chúa Thánh Thần

04. Tuần Cửu Nhật Cầu Xin Lòng Thương Xót Chúa

05. Đọc 1 Kinh lay Cha, 1 Kinh Kinh Mừng, 1 Kinh Tin Kinh

06. Chuỗi Hạt Lòng Thương Xót...

07. Hát Kính Lòng Thương Xót Chúa

08. Kinh Phó Thác Cho Đức Mẹ Khiết Tâm

09. Kinh Cầu Nguyện Cho Sự Sống

10. Kinh Cầu Cho Các Linh Mục

11. Thánh ca dâng lên Chúa

12. Phúc Âm và Suy Niệm Lời Chúa (nếu có cha)

12.1. Phúc Âm và 12.2. Suy Niệm

13B. Kinh Cầu Cho Các Bệnh Nhân (nếu có)

13H. Cầu Xin Cho Người Hấp Hối (nếu có)

14. Kinh Rước Lễ Thiêng Liêng 1 hoặc 2

15A. Kinh Bời Lời

15B. Kinh Vực Sâu

16. Thánh $\mathrm{Ca}$ Cầu Hồn

17. Kinh Cám ơn

18. Kinh Trông Cậy, Các Câu Lạy, Làm Dấu Thánh Giá

19. Thánh Ca Dâng lên Mẹ Maria

19a. Cha ban phép lành (nếu có cha)

20. Lời cám ơn kết thúc, mời sinh hoạt 


\section{Anh-Ngoc HOANG}

Fig.7 - Indications sur le site Mekhiettam.org pour réciter la prière à la divine miséricorde.

En fait, ce dispositif numérique catholique propose non seulement des prières "virtuelles ", mais aussi des activités caritatives «dans la vie réelle » à ses membres, tel qu'il est formulé dans sa "vision » [Tôn Chi]. Concrètement, depuis sa fondation en 2009, à chaque carême ${ }^{\dagger i}$, la " famille Mekhiettam ", comme ses membres s'autodésignent, met en œuvre le "programme Ephata» qui consiste à recueillir des fonds consacrés aux activités caritatives au Việt Nam faveur "des orphelins, des pauvres et des personnes âgées solitaires ". Outre des dons volontaires classiques, ce groupe catholique vietnamien propose également un programme de collecte de fonds spécifique intitulé " programme de chansons sur demande contre offrande " ("Chương trinh nhạc bảo trợ") : les gens remettent un don en contrepartie duquel ils demandent que soient chantés des chants (le plus souvent catholiques) en leur nom.

L'intérêt de ce phénomène sous l'angle de notre analyse est la diversité des possibilités de participation et d'engagement des internautes membres de ce salon à cette activité. En fait, certains membres offrent leur service en chantant des chansons sur demande, d'autres participent effectivement au voyage au Việt Nam afin de mettre en œuvre localement leurs actions caritatives dans différents lieux au Việt Nam. Sont publiés sur le site Mekhietam de nombreux détails du " programme Ephata » annuel de la première à la cinquième édition.

$\mathrm{Si}$ ces informations reflètent une prise de conscience du principe de transparence important dans ce domaine, elles nourrissent également un sentiment d'appartenance à une même

11 Temps de pénitence pour les catholiques, qui dure depuis le mercredi dit « des cendres » jusqu'au jour de Pâques.

tic\&société - 9 (1-2), 2015 
La foi catholique et les dispositifs numériques:

le cas de la diaspora catholique vietnamienne

communauté. Par ailleurs, outre ce programme annuel de charité, ils ont également partagé d'autres pratiques religieuses en face-àface comme un pelerinage en Terre Sainte, une retraite spirituelle à Berlin, ou même des événements humains et sociaux tels que le mariage d'un membre ${ }^{12}$. Entre eux, un fort sentiment d'attachement mutuel est né : "Nous nous considérons plus que frères et sœurs pourvus des liens du sang ", nous disait $\mathrm{M}^{\mathrm{me}}$ Lan. Une bonne illustration de ces liens spirituels et interpersonnels établis entre les membres de la "famille Me Khiet tam" pourrait être la page nommée « activités de la vie » [Sinh hoạt] ${ }^{13}$ montrant des photos de cette " famille " au $2^{\mathrm{e}}$ anniversaire de ce salon de discussion en 2011. Ce qui semble être montré ici n'est pas un "groupe virtuel » avec des avatars, mais " des visages humains réels " engagés dans les interactions «réelles». "Réel » est également un autre effet de cette communauté catholique vietnamienne hybride: la conversion au catholicisme de deux internautes qui la fréquentaient, selon $\mathrm{M}^{\mathrm{me}} \mathrm{Lan}$.

Ainsi le dispositif numérique Me khiet tam (« Notre Dame du Cœur pur ») permet de former une communauté émergente et hybride de foi catholique. En effet, en commençant par «prier ensemble en ligne ", ces usagers d'internet qui sont des catholiques vietnamiens dispersés partout dans le monde en sont venus à « agir ensemble dans la vraie vie - IRL [in real life] 》 à travers des actions caritatives, des pratiques de foi traditionnelles (pèlerinage, retraite, formation, etc.), pour enfin former une «famille» spirituelle qui se nourrit aussi bien des choses religieuses que des liens humains et qui «porte du fruit » (succès du programme caritatif, travail d' " évangélisation » auprès de nonchrétiens).

Mais ce cas ne semble pas exceptionnel, il montre plutôt une nouvelle réalité des catholiques vietnamiens contemporains, en

${ }_{12}^{12}$ Ces informations ont été communiquées par $M^{\text {me }}$ Lan pendant l'entretien.

13 <http://mekhiettam.org/home/?a=shoat>, dernière consultation le 5 novembre 2014. 


\section{Anh-Ngoc HOANG}

particulier ceux d'outre-mer. En effet, ces derniers ont recours à des dispositifs numériques pour leurs pratiques religieuses catholiques, comme ils l'ont confirmé dans notre questionnaire. Plus des trois-quart d'entre eux $(78 \%)$ étaient d'accord avec cette proposition: "Les médias numériques apportent une aide aux chrétiens qui ne peuvent pas aller à l'église pour des raisons de santé, d'incarcération, de travail, d'éloignement géographique ». En général, la majorité d'entre eux (67\%), estime que "deux univers, celui du "média numérique" et celui de "la vie réelle" sont complémentaires l'un de l'autre ", même s'ils ont nuancé en précisant que "des sites web catholiques ne sont que complémentaires à la vie de foi. L'essentiel reste la participation à la prière et le vécu des sacrements. » (76\%).

\section{Conclusion}

Au stade actuel de notre recherche, les premiers résultats ont montré que des catholiques vietnamiens d'outre-mer façonnent l'imaginaire d'un catholicisme contemporain qui reflète une tentative d'intégrer des pratiques religieuses online et offline afin de vivre au mieux leur foi à l'ère de la mobilité croissante et de la technologie omniprésente. Cette réalité émergente pose de nouveaux défis mais ouvre aussi de nouvelles perspectives pour l'Église catholique en général. Même s'il parait réducteur de concevoir l'Église comme un pur " hub de connexions ", formé de formes relationnelles « liquides ", il devient significatif de penser la foi dans son rapport au monde d'aujourd'hui, en s'interrogeant, par exemple, comme l'a fait le théologien jésuite Antonio Spadaro "Est-il possible de, penser à internet comme à une métaphore pour comprendre l'Église, sans croire pour autant, naturellement, qu'elle puisse être exhaustive ? " (Spadaro, 2012, p.63). Et notre travail d'investigation semble confirmer en partie l'une de ses réflexions : " l'Eglise au temps des réseaux sociaux est appelée à une tâche de ce genre, en prenant les formes adaptées, mais s'entendant aussi (et donc certainement non seulement) comme un lieu de connexion significative des personnes, capable de

tic\&société - 9 (1-2), 2015 
La foi catholique et les dispositifs numériques:

le cas de la diaspora catholique vietnamienne

fournir la base pour construire des rapports de communion dans une société fragmentée » (idem, pp.65-66).

Enfin, l'objectif scientifique de ce travail ambitionne d'identifier une dynamique imaginative à l'œuvre dans le processus de l'articulation du religieux et du numérique à l'ère de la globalisation. Ainsi le choix qui est posé dans cette enquête et qui consiste à appréhender les rapports entre la population diasporique catholique vietnamienne et les Tic est motivé par la volonté de comprendre cette problématique vietnamienne particulière en l'inscrivant dans un phénomène contemporain plus large " porteur d'enjeux essentiels ", dans " une question qui est au cœur du devenir du monde contemporain » (Mattelart, 2009 a), un monde, comme l'indique Dana Diminescu (2010, p.10) « atteint par une mobilité généralisée et par une amplification sans précédent de la communication ».

\section{Références bibliographiques}

APPADURAI A., 2001, Après le colonialisme: les conséquences culturelles de la globalisation, trad. F. Bouillot, Paris, Payot.

ARASA D., L. CANTONI et L. A. RUIZ (dir), 2010, Religious Internet Communication. Facts, Trends and Experiences in the Catholic Church, Roma, Edusc.

CAMPBELL H., 2013, "Community" dans H. A. CAMPBELL (dir), Digital Religion. Understanding Religious Practice in New Media Worlds, New-York, Routledge, pp.57-71.

COUTANT A. et J.-C. DOMENGET, 2014, «Un cadre épistémologique pour enquêter sur les dispositifs sociotechniques d'information et de communication " dans H. BOURDELOIE et 


\section{Anh-Ngoc HOANG}

D. DOUYÈRE (dir), Méthodes de recherche sur l'information et la communication, Paris, Mare et Martin, pp.231-253.

DAVALLON J., 2004, «Objet concret, objet scientifique, objet de recherche », Hermès, n³8, pp.30-37.

DELAUNAY A., "Communion", dans Encyclopædia Universalis, <http://srvext.uco.fr:2062/encyclopedie/communion/>, dernière consultation le 20 novembre 2014.

DIMINESCU D., 2010, “Présentation », Réseaux, vol.28, $\mathrm{n}^{\circ} 159$, Les migrants connectés, T.I.C., mobilités et migrations, pp.9-13.

ĐÕ Hữu Nghiêm A., NGUYẼ̃N Ngọc Sơn A., TRÀN Qúy Thiện J., 2004, "Cộng đồng Công giáo Việt Nam hải ngoại " [Communautés diasporiques des catholiques vietnamiens], dans Giáo hội Công giáo Việt Nam. Niên giám 2004 [Eglise catholique vietnamienne. Annuaire de 2004], Hà Nội, Nhà xuất bản Tôn giáo.

DORAIS L.-J., 2007, "Faith, Hope and Identity: religion and the Vietnamese Refugees", Refugee Survey Quarterly, vol.26, $\mathrm{n}^{\circ} 2$, pp.57-68.

DORAIS L.-J., 2001, «Defining the Overseas Vietnamese ", Diaspora, vol.30, $\mathrm{n}^{\circ} 1$, pp.3-27.

DOUYERE D., 2010, "Communication et religion chrétienne catholique », Cahiers de la Sfsic, n5, pp.17-18.

DUFOIX S., 2011, La dispersion. Une histoire des usages du mot diaspora, Paris, éditions Amsterdam.

FLINN F., 2007, Encyclopedia of Catholicism, New York, Checkmark Books.

HELLAND C., 2013, "Ritual" dans H. A. CAMPBELL (dir), Digital Religion. Understanding Religious Practice in New Media Worlds, New-York, Routledge, pp.25-40.

tic\&société - 9 (1-2), 2015 
La foi catholique et les dispositifs numériques:

le cas de la diaspora catholique vietnamienne

HOANG A.-N. et C. VIGNE, 2014, "Vietnam », dans G. SIMON (dir), Dictionnaire géo-historique des migrations internationales, Paris, Armand Colin.

HÜWELMEIER G., 2014, "Performing Intimacy with God: Spiritual Experiences in Vietnamese Diasporic Pentecostal Networks », German History, vol.32, n॰3, pp.414-430.

KAWAKAMI I., 2003, "Resettlement and Border Crossing: A Comparative Study on the Life and Ethnicity of Vietnamese in Australia and Japan", International Journal of Japanese Sociology, vol.12, $\mathrm{n}^{\circ} 1$, pp.48-67.

LE Dinh Bang, 2009, Hành Trình 100 Năm Báo Chí Công Giáo Việt Nam [Parcours centenaire de la presse catholique vietnamienne], Hà Nội, NXB Tôn Giáo.

LE MAREC, J., 2002, Ce que le "terrain » fait aux concepts: vers une théorie des composites, habilitation à diriger des recherches, Université Paris 7.

LUNDBY K., 2013, «Theoretical Frameworks for Approaching religion and new media ", dans H. A. CAMPBELL (dir), Digital Religion. Understanding Religious Practice in New Media Worlds, New-York, Routledge, pp.225-237.

MARECHAL D., C. MÉADEL et I. VEYRAT-MASSON, 2011, "Présentation », Le Temps des Médias, ${ }^{\circ} 17$, Communiquer le sacré, pp.6-10.

MATTELART T., 2009a, "Présentation », tic \& société, vol.3, $\mathrm{n}^{\circ} 1-2,<\mathrm{http}: / /$ ticetsociete.revues.org/612>, dernière consultation 8 avril 2014.

MATTELART T., 2009b, «Les diasporas à l'heure des technologies de l'information et de la communication: petit état des savoirs ", tic \& société, vol.3, $n^{\circ} 1-2$, <http://ticetsociete.revues.org/600>, dernière consultation le 9 avril 2014. 


\section{Anh-Ngoc HOANG}

MONNOYER-SMITH L., 2008, «Pour une épistémologie complexe des SIC ", XVI ${ }^{e}$ congrès de la Société française des sciences de l'information et de la communication (Sfsic), Compiègne, <http://www.sfsic.org/congres 2008/spip.php?article78>, dernière consultation le 3 décembre 2015.

NGOC LAN, 2010, "Truyền thông Công giáo Việt Nam trong 50 năm qua" [Communication catholique vietnamienne depuis 50 ans], <http://www.hdgmvietnam.org/truyen-thong-cong-giao-taiviet-nam-trong-50-nam-qua/1777.65.24.aspx>, dernière consultation le 29 août 2014.

NGUYEN Ngoc Son, 2006, "Vài Nét Về Hiện Trạng Truyền Thông Xã Hội Tại Việt Nam » [Quelques remarques sur la réalité de la communication sociale au Viêt Nam], $<$ http://www.thanhlinh.net/node/4667> $>$, dernière consultation le 29 août 2014.

PHAN P. C., 2000, "Vietnamese Catholics in the United States: Christian Identity between the Old and the New", U.S Catholic Historian, vol.18, n 1, Asian-American Catholics, pp.19-35.

SPADARO A., 2014, Cyberthéologie, penser le christianisme à I'heure d'internet [2012], trad. C. De Paepe, J.-M. Faux et $\mathrm{H}$. Jacobs, Bruxelles, Lessius.

TRAN Van Toan A., 1998, "Catholiques vietnamiens en France ou le retour de l'inculturé ", dans M. SPINLER et A. LENOBLEBART (dir), Chrétiens d'outre-mer en Europe : un autre visage de l'immigration, Karthala, pp.147-164.

Vatican, Conseil pontifical pour les communications sociales, 1963, "Inter Mirifica », $<$ http://www.vatican.va/archive/hist councils/ii vatican council/doc uments/vat-ii decree 19631204 inter-mirifica fr.html>, dernière consultation le 31 mars 2014.

Vatican, Conseil pontifical pour les communications sociales, 1971 , «Communion et Progrès », 
La foi catholique et les dispositifs numériques :

le cas de la diaspora catholique vietnamienne

<http://www.vatican.va/roman curia/pontifical councils/pccs/docu ments/rc pc pccs doc 23051971 communio fr.htmls dernière consultation le 31 mars 2014.

Vatican, Conseil pontifical pour les communications sociales, 2002, "L'Eglise et Internet ", $<$ http://www.vatican.va/roman curia/pontifical councils/pccs/docu ments/rc pc pccs doc 20020228 church-internet fr.html>, dernière consultation le 31 mars 2014. 